\title{
Relación entre los comportamientos no lineales dieléctrico y mecánico en cerámicas piezoeléctricas de PZT
}

\author{
R. PÉREZ, A. ALBAREDA, J.E. GARCíA, J.A. CASALS \\ Departament de Física Aplicada. Universitat Politècnica de Catalunya. \\ Jordi Girona 1-3, Campus Nord. 08034 Barcelona.
}

\begin{abstract}
La aplicación de campos eléctricos o esfuerzos mecánicos elevados sobre piezocerámicas PZT produce una alteración de los coeficientes elásticos y dieléctricos, así como de sus pérdidas. En este trabajo se establece un procedimiento que permite la comparación entre el comportamiento no lineal dieléctrico de una cerámica con el comportamiento no lineal elástico medido en el primer modo de oscilación radial, describiéndolos mediante magnitudes adecuadas. Se observa un comportamiento cualitativamente distinto entre los materiales PZT blandos y duros. Para un mismo material, los comportamientos dieléctrico y mecánico son claramente semejantes, así como las pérdidas de uno y otro tipo. Esto se puede interpretar si se asume que la no linealidad se produce gracias a la interacción de las paredes de dominio con los defectos, y si solo intervienen apreciablemente las paredes de no- $180^{\circ}$. Se analizan diversos factores a tener en cuenta en esta comparación, como son el tipo de no linealidad, la distribución de las orientaciones de los dominios, o la falta de uniformidad del campo. Ello puede permitir relacionar el comportamiento no lineal con la función de distribución (que define el estado de la cerámica) o la interpretación del comportamiento de los modos superiores.
\end{abstract}

Palabras clave: piezoeléctrico, cerámica, no linealidad, dieléctrico, elasticidad.

Relation between dielectric and mechanical nonlinear behaviours in PZT piezoelectric ceramics.

Elastic and dielectric coefficients of PZT piezoceramics are perturbed by applying high electric field or high mechanical stress. In this work, a procedure is described in order to compare non-linear dielectric and elastic behaviour, by using suitable quantities. Qualitative differences are observed between soft and hard materials. But, for a given material, dielectric and mechanic behaviours are very similar, as well as the losses of both kinds. This fact can be understood by assuming that non-linearity is produced by interaction between domain walls and defects, and that $180^{\circ}$ walls are negligible in front of non $-180^{\circ}$ walls. Some facts must be taken into account in order to compare both behaviours, as the non-linear model, the domain wall orientation distribution, or the non uniformity of the fields. This allows to relate the non linear behaviour with the distribution function, that defines the material state, or to understand the non-linearity produced at the overtones.

Key words: piezoelectric, ceramics, non-linearity, dielectrics, elasticity.

\section{INTRODUCCIÓN}

Las cerámicas piezoeléctricas se han hecho insustituibles en muchos dispositivos de potencia en los que se requiere un buen factor de acoplo electromecánico, pero cuando se requieren además máximas prestaciones hay que usar dichas cerámicas en condiciones límite. Desafortunadamente, su comportamiento en estas condiciones es altamente no lineal, siendo sus características fuertemente dependientes del campo aplicado. Por ello, es necesario no solo descubrir el origen interno de dicho comportamiento, lo que nos permitiría posiblemente el diseño de cerámicas con prestaciones mejores y prevenir los efectos negativos del envejecimiento y la fatiga, sino también la caracterización a alta señal a fin de poder optimizar el diseño de los dispositivos.

Varios métodos han sido descritos en los últimos años para su caracterización no lineal dieléctrica $(1,2)$, piezoeléctrica $(3,4)$ o elástica $(4,5,6)$, y se han observado notables coincidencias entre dichos aspectos, tanto a nivel de la caracterización lineal como no lineal, observando como se alteran los coeficientes correspondientes (7) o las pérdidas (8).

Para describir o explicar dichos comportamientos se han desarrollado varias teorías, inspiradas a menudo en modelos usados en ferromagnetismo como los de Rayleigh o Preisach $(9,10)$. En ellas se reco- noce como causa fundamental de dichas anomalías a la interacción de las paredes de dominio de no- $180^{\circ}$ con los centros de anclaje. Por ello, aun cuando el comportamiento lineal tiene una componente intrínseca (debida a la deformación de la red cristalina) y otra extrínseca (debida a otras causas como el movimiento de paredes de dominios), se asume que la no linealidad es producida solamente por esta última causa.

En este estudio se pretende comparar los fenómenos no lineales producidos en dos circunstancias distintas: en primer lugar, aplicando un esfuerzo $T$ elevado, excitando eléctricamente un modo de resonancia radial, a fin de observar la no linealidad elástica, y en segundo lugar, aplicando un campo eléctrico $E$ elevado, a baja frecuencia, con lo que se puede analizar la no linealidad dieléctrica. El objetivo consiste en mejorar la comprensión de los mecanismos que causan la no linealidad y las pérdidas, a la vez que se puede facilitar, desde un punto de vista totalmente empírico, la caracterización no lineal necesaria para el diseño dispositivos de potencia o para comprobar su estado de envejecimiento. Dada la mayor simplicidad de la medida en baja frecuencia, que no precisa de ningún requisito especial tal como ocurre en resonancia, si se reconocen suficientes paralelismos entre los dos comportamientos, se facilita la caracterización de la cerámica al permitir la elección del método de caracterización. 


\section{ANÁLISIS TEÓRICO}

Asumiendo que la no linealidad proviene del efecto extrínseco (11), existen razones para pensar que debe observarse un comportamiento similar en las dos situaciones. Para ello se analiza la dinámica del desplazamiento de una determinada pared de dominio $(12,13)$. Podemos establecer un sistema de coordenadas ligado a la pared de dominio, tal como se muestra en la figura 1 . En ella, el vector de polarización espontánea es siempre perpendicular a la dirección 3. Para que los dos dominios que confluyen en una pared sean compatibles cristalográfica y eléctricamente (14) es necesario que la componente del campo $D_{1}$ perpendicular a la pared, sea continua, por lo que el campo $E_{1}$ no puede favorecer ninguno de los dos dominios. Tanto si la pared de dominio es de $180^{\circ}$ como si no, solo la componente $E_{2}$ es capaz de producir un desplazamiento de la pared. Si la pared no es de $180^{\circ}$, ésta será sensible a la aplicación de un esfuerzo de cizalladura $T_{12^{\prime}}$, pero no a ninguna otra componente, mientras que si es de $180^{\circ}$ no existirá ni esta posibilidad.

$\mathrm{Al}$ producirse un desplazamiento $\delta$ de la pared (en la dirección 1), el vector desplazamiento eléctrico medio alterará su componente $D_{2}$. Si la pared no es de $180^{\circ}$, alterará además el valor medio de $S_{12}$. Para una $\delta$ dada los incrementos de $D_{2}$ y de $S_{12}$ están perfectamente determinados, y dependen exclusivamente de las características de la celda elemental, pudiendo depender por tanto de la estructura tetragonal o romboédrica, pero no de los defectos. Esto conlleva que, si solamente estuviera presente el movimiento de paredes de dominio distintas de $180^{\circ}$, la constante de acoplamiento electromecánica seria la unidad.

Si los fenómenos no lineales y de aumento de pérdidas dependen de la interacción de la pared con los centros de anclaje, estas interacciones se producirán en función de $\delta$, no importa cual sea el origen, eléctrico o mecánico, de dicho movimiento. Por ello, si las paredes no son de $180^{\circ}$, se espera que la no linealidad sea similar en ambos casos, lo que no debe ocurrir con paredes de $180^{\circ}$ ni tampoco con el efecto intrínseco.

La dependencia entre $\delta$ y el campo aplicado $E_{2}$ o $T_{12}$, puede ser lineal o no, siguiendo en este último caso alguno de los modelos previstos. Se deduce que el modelo a seguir debe ser común para el efecto elástico y el efecto dieléctrico; pero, a pesar de ello, existen razones para pensar que el comportamiento no puede ser idéntico. Por un lado, parte de los efectos observados pueden provenir del desplazamiento de paredes de $180^{\circ}$ o del efecto intrínseco y, por otra parte, los dominios no se orientan todos de igual forma respecto del disco, dando lugar a una cierta distribución que depende de las orientaciones de

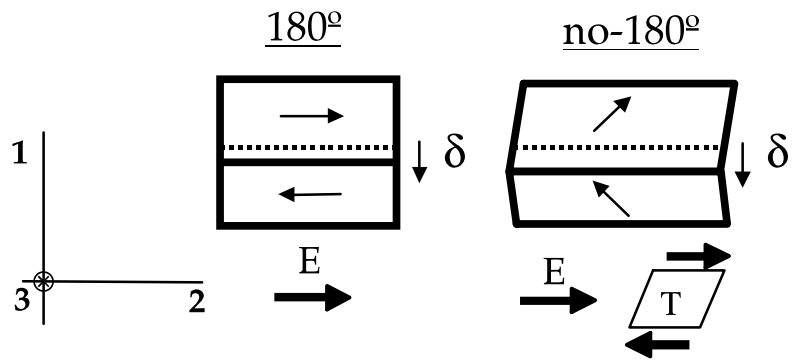

Figura 1: Coordenadas asociadas a la pared del dominio. Componentes de $E$ y $T$ que pueden producir un desplazamiento de la pared, tanto para las paredes de $180^{\circ}$ como de no- $180^{\circ}$

los dominios. Además, el orden de los tensores dieléctrico y elástico son distintos, y por lo tanto se transforman de manera distinta al pasar de un sistema de referencia a otro. Por último, se debe considerar la incidencia de la no uniformidad de los campos aplicados.

\section{MEDIDA DE LA NO LINEALIDAD}

\subsection{No linealidad elástica}

La medida de la no linealidad elástica se lleva a término mediante la aplicación de una tensión eléctrica, a una frecuencia próxima a la frecuencia de resonancia $(5,15,16)$ durante un tiempo suficiente para que se alcancen las condiciones estacionarias. Para una misma frecuencia se aplican tensiones de distinta amplitud y se mide la impedancia en cada caso, así como la corriente total. Mediante una medida previa se determina el valor de la capacidad $C^{\text {s }}$, lo que nos permite deducir la corriente "motional" (proporcional al desplazamiento eléctrico medio) y la impedancia $Z_{\mathrm{m}}$ de la parte motional.

Si el sistema fuera lineal, la resistencia seria constante y la reactancia dependería linealmente de la frecuencia. Sin embargo se observa como ambas se incrementan con el aumento de la corriente, denotando un aumento de las pérdidas y un desplazamiento de la frecuencia de resonancia. En la figura 2 se observa como dichos incrementos son independientes de la frecuencia de la señal aplicada. También se aprecia una dependencia de la reactancia $X$ con la corriente $I$ que puede ser lineal o cuadrática dependiendo del tipo de material. Se demuestra que el incremento de la reactancia, $\Delta X$, es proporcional a la alteración relativa del coeficiente elástico $\Delta s / s(17)$.
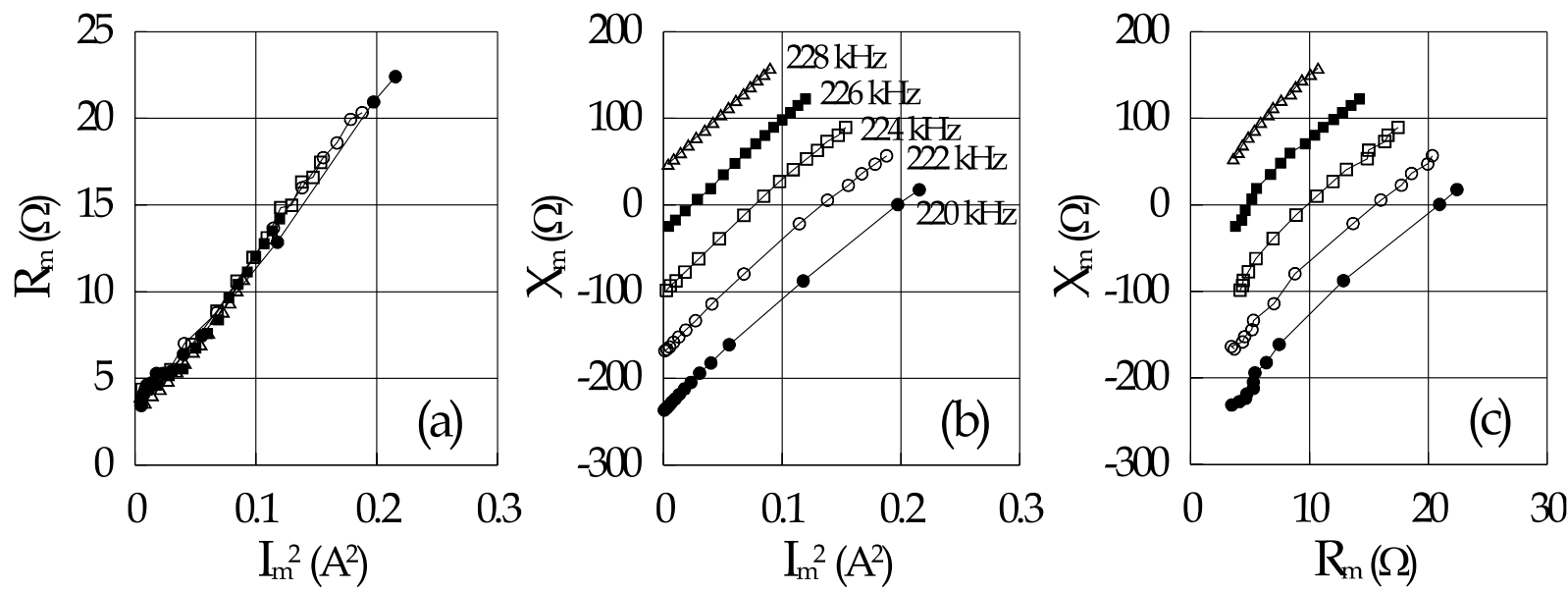

Figura 2: No linealidad elástica: resistencia (a) y reactancia (b) en función del cuadrado de la corriente para varias frecuencias próximas a la resonancia $(226.8 \mathrm{kHz})$. En (c): correlación entre la resistencia y la reactancia representadas en (a) y (b). 


\subsection{No linealidad dieléctrica}

Las medidas dieléctricas se realizan a la frecuencia de $1 \mathrm{kHz}$, muy inferior a la primera frecuencia de resonancia, aplicando campos sinusoidales intensos, de distinta amplitud y registrando la corriente no lineal, a la que se ha substraído, previamente, la parte lineal mediante un puente adecuado (2). Integrando dicha corriente en función del tiempo se obtiene la parte no lineal del vector desplazamiento eléctrico $D$. Esta contribución se separa en dos partes: una que depende solo de $E$ pero no del signo de su derivada temporal, que se corresponde con la parte reversible, y otra que depende de dicho signo, que se corresponde con la parte irreversible. De cada una de dichas partes se obtienen los valores de la parte real e imaginaria de la permitividad, $\varepsilon^{\prime}$ y $\varepsilon^{\prime \prime}$, respectivamente, que pueden relacionarse con la amplitud del campo aplicado $(2,18,19)$.

Se obtiene una cierta relación lineal o cuadrática de $\varepsilon^{\prime}$ con el campo aplicado, que depende del tipo de PZT, y una relación lineal entre las partes real e imaginaria de la permitividad, ya que ambas dependen de $E$ de la misma forma (figura 3 ).

\subsection{Comparación entre ambos comportamientos}

Para poder comparar los comportamientos no lineales dieléctricos y elásticos de una determinada muestra se precisa describir ambos utilizando las mismas magnitudes. Por ello es poco adecuado describirlos en función del campo $E$ o del esfuerzo $T$, puesto que en las experiencias dieléctricas $T$ es prácticamente nulo, mientras que en las elásticas se anula el campo $E$. Seria ideal poder utilizar como variable el desplazamiento $\delta$ de las paredes, pero esta es una magnitud inaccesible experimentalmente, siendo distinta en cada grano. Debido a la relación unívoca entre $\delta$ y el desplazamiento eléctrico $D$, podemos usar el valor medio de ésta como variable independiente, obtenida de manera directa de la medida de la intensidad de corriente.

Sin embargo, en el caso elástico, se observa que para obtener una alteración de los coeficientes independiente de la frecuencia se requiere tomar solamente la parte "motional" de $D$, deduciendo la parte eléctrica a partir del valor de $\varepsilon^{\mathrm{S}}$ obtenido a alta frecuencia.

A fin de comparar la alteración de los coeficientes reales $\mathrm{s}$ y $\varepsilon$ a causa de la amplitud del campo se podrán usar la magnitudes adimensionales $\Delta s / s$ y $\Delta \varepsilon / \varepsilon$. Para comparar las pérdidas, se usa la energía perdida $\eta$ a lo largo de un ciclo por unidad de volumen. Debido a la fuerte dependencia con $D$, se prefiere utilizar $\eta / D^{2}$. Debe observarse que, en el caso de que la tangente de pérdidas fuera constante, $\eta / D^{2}$ no dependería de la amplitud de $D$.
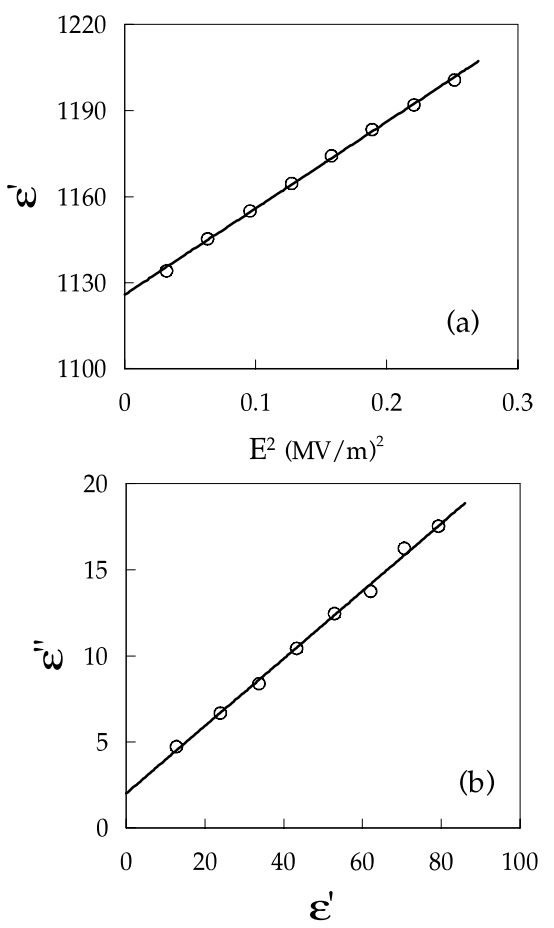

Figura 3: No linealidad dieléctrica: (a) relación entre la constante dieléctrica y el cuadrado de la amplitud del campo eléctrico a $1 \mathrm{kHz}$; (b) relación entre la parte imaginaria de la constante dieléctrica y el incremento de la parte real de la misma.

La densidad de energía mecánica perdida por ciclo $\eta_{\text {mec }}$ se obtiene a partir del valor eficaz de la corriente motional $I_{\mathrm{m}}(\mathrm{rms})$, a partir la de expresión:

$$
\eta_{\text {mec }}=\frac{I_{m}{ }^{2} \cdot R_{m}}{V \cdot f}
$$

donde $R_{\mathrm{m}}$ es la resistencia motional, $V$ el volumen de la muestra y $f$ es la frecuencia de la señal sinusoidal aplicada. La correspondiente magnitud dieléctrica, $\eta_{\text {elec }}$ se obtiene a partir del valor eficaz del campo eléctrico aplicado:

$$
\eta_{\text {elec }}=2 \pi \cdot \varepsilon_{0} \varepsilon^{\prime \prime} \cdot E_{\text {rms }}^{2}
$$

En la figura 4 se observa claramente un comportamiento cualitativamente distinto entre blandos y duros. Mientras que en los materiales duros o muy duros, tanto en la parte lineal como en imaginaria, las funciones son cóncavas, con unos valores casi constantes a baja am-
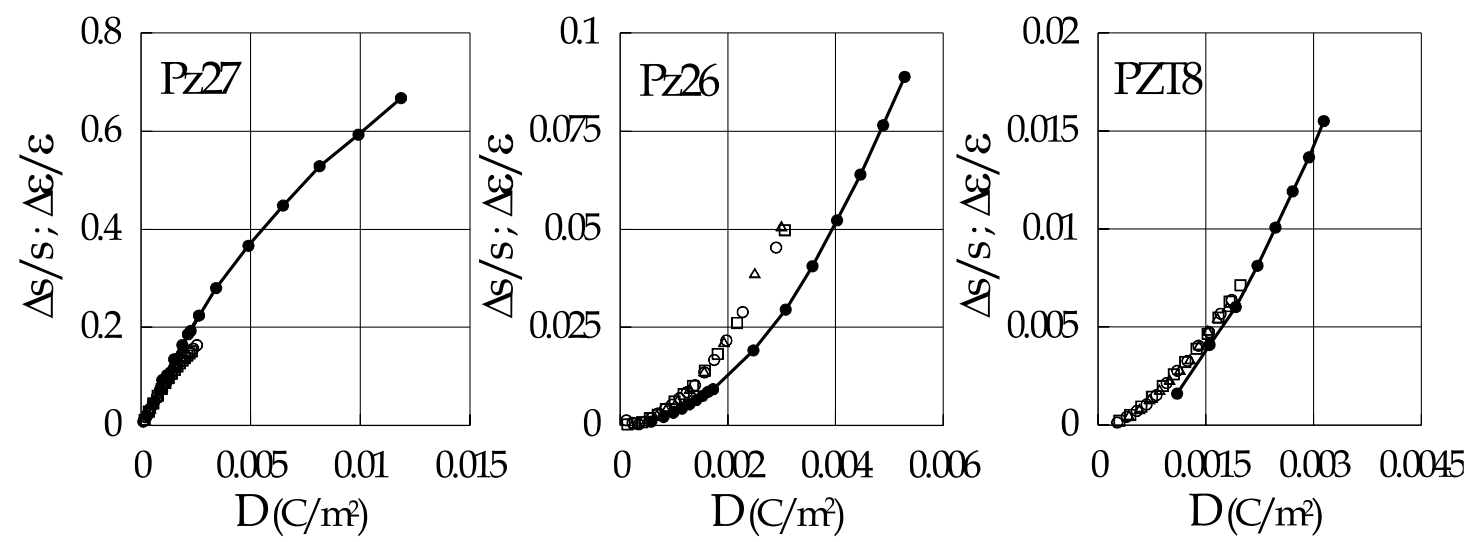

Figura 4: Relación entre el incremento relativo de la constante dieléctrica $\Delta \varepsilon / \varepsilon(\bullet)$ y el incremento relativo de la constante elástica $\Delta s / s$ (símbolos sin relleno), en función de $D$ para tres materiales distintos: blando (Pz27), duro (Pz26) y muy duro (PZT8). La constante elástica se ha representado a tres frecuencias distintas, próximas a la frecuencia de resonancia de la muestra. 

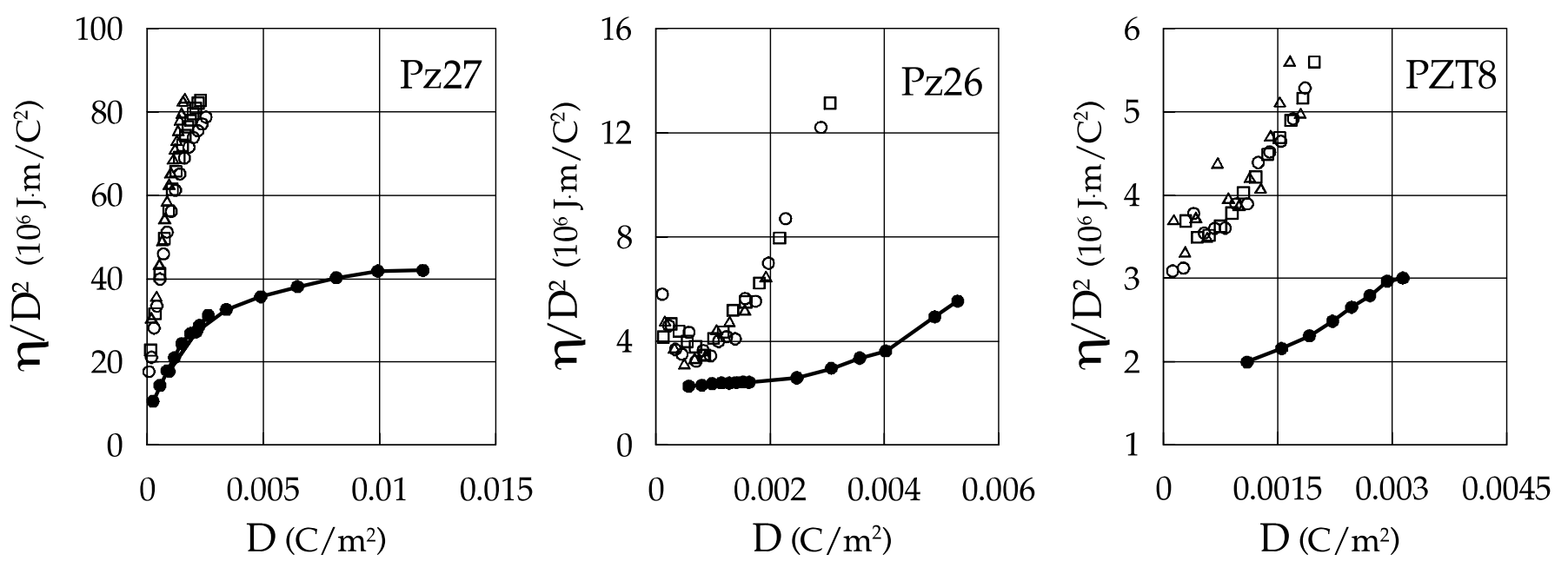

Figura 5: Relación entre las pérdidas dielectricas $(\bullet)$ y mecánicas (símbolos sin relleno), expresadas como $\eta / D^{2}$, en función de la amplitud de $D$, para tres materiales distintos: blando (Pz27), duro (Pz26) y muy duro (PZT8). La pérdidas elásticas se han representado a tres frecuencias distintas, próximas a la frecuencia de resonancia de la muestra.

plitud, en los materiales blandos son convexas, manifestando un comportamiento lineal a amplitudes bajas. De manera análoga se procede con $\eta / D^{2}$, obteniendo unas curvas que tienen cierta semejanza con las obtenidas al comparar los coeficientes reales (figura 5).

En general, se observa una buena concordancia cuantitativa entre la alteración de los coeficientes $s$ y $\varepsilon$ (figura 4), aunque las pérdidas elásticas son notablemente mayores que las pérdidas dieléctricas, en la representación usada (figura 5).

\section{ANÁLISIS DE LOS FACTORES QUE INFLUYEN EN EL COMPORTAMIENTO NO LINEAL}

Si bien en el análisis teórico se han establecido las condiciones necesarias para que haya concordancia entre los resultados elásticos y dieléctricos, existe un conjunto de factores que no solo condicionan dicho comportamiento, sino que pueden condicionarlo de manera distinta en cada caso.

El primer hecho a considerar es que solo se puede esperar una buena correspondencia si no intervienen los efectos intrínseco ni el movimiento de paredes de $180^{\circ}$. Si se asume que los efectos intrínsecos son totalmente lineales, este factor no debe ser causa de divergencia entre ambos resultados, siendo su único efecto el que una parte del desplazamiento eléctrico $D$ no contribuye a la no linealidad. En cambio, las paredes de $180^{\circ}$ producirían una diferencia notable. El hecho de que no aparezca esta diferencia nos conduce a que la cantidad de paredes de este tipo debe ser demasiado pequeña, en términos relativos. La buena correspondencia entre los coeficientes dieléctricos y piezoeléctricos avala dicha hipótesis. (7)

En segundo lugar, debemos tener en cuenta que las paredes de dominio están orientadas, según cada grano, en una dirección distinta (8). Tal como han propuesto algunos autores (20), se precisa utilizar un tratamiento estadístico. Se puede definir una función de distribución de forma que a cada orientación relativa de los ejes de los dominios respecto a los ejes del disco, definida mediante los ángulos de Euler, se le hace corresponder la superficie total de pared existente en un volumen dado, para cada tipo de pared. Esta función de distribución dependerá de los tratamientos realizados sobre la cerámica, por lo que el tamaño de grano, los tratamientos térmicos, la polarización, la compresión, el envejecimiento, la fatiga, etc., influirán sobre dicha distribución. Algunos de estas acciones romperán la simetría de la función de distribución y harán posible que el material pueda tener ciertas propiedades a nivel macroscópico. Esto es cierto no solo para el efecto piezoeléctrico lineal, sino también para ciertas propiedades no lineales. No tenemos una manera directa de conocer dicha distribución, pero un buen conocimiento de los mecanismos que producen el desplazamiento de paredes podría permitirnos conocer, a través de la medida del comportamiento lineal y no lineal, algunas características de ella. El problema de la dispersión de orientaciones viene agravado por el hecho de que los distintos granos no se pueden considerar independientes, ya que cualquier alteración eléctrica o mecánica de uno de dichos granos repercute en sus vecinos, tanto por la vía eléctrica como mecánica, de tal forma que depende de la orientación relativa entre ellos. Como primera aproximación, es sensato no considerar dicho efecto de interacción, asumiendo el error que con ello se introduce.

En tercer lugar, debe tenerse en cuenta el orden de los tensores que intervienen, que afectará de manera distinta a las distintas variables en el momento de obtener $E_{2}$ o $T_{12}$ a partir de los campos $E$ y $T$ macroscópicos, así como para obtener $D$ y $S$ macroscópicos a partir de $D_{2}$ o $S_{12}$ de cada pared de dominio (figura 6). Por otra parte, la relación no lineal entre los campos y el desplazamiento de la pared no es la misma para todos los tipos de material, por lo que dichas divergencias serán mayores cuanto mayor sea el orden de la dependencia no lineal.

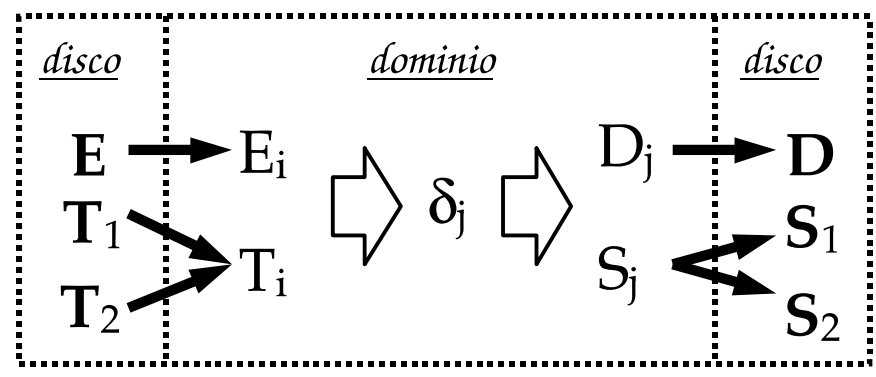

Figura 6: Contribución de un determinado dominio en la obtención de los tensores $D$ y $S$ en un punto de la muestra, en función de los campos macroscópicos locales $E$ y $T$ aplicados. 
Por último, cabe comentar el hecho de que, mientras que en las experiencias a baja frecuencia el campo es uniforme, no es así en resonancia y mucho menos si se consideran los modos superiores. Si bien la no linealidad aparecerá a consecuencia del valor local del campo $D$, todas las relaciones establecidas experimentalmente se refieren al valor medio del campo $D$, integrado a lo ancho del electrodo. Esto no representa ningún obstáculo para los efectos lineales, pero sí para un efecto no lineal. Si suponemos que hay una dependencia potencial con exponente $\alpha$, podemos definir un factor correctivo $g=\left\langle D^{\alpha}\right\rangle \mid\langle D\rangle^{\alpha}$, siendo $<D>$ y $<D^{\alpha}>$ los valores medios de $D$ y $D^{\alpha}$ extendidos al volumen de la muestra, que da la relación entre la magnitud que usamos y la que deberíamos usar. Aunque este factor es cercano a la unidad para el primer modo, es mucho mayor en los modos superiores.

Se ha efectuado la medida de la no linealidad para distintos modos radiales de vibración sobre un mismo disco. Como se observa en la figura 7, para que los resultados sean coincidentes es necesario corregir el valor medio de $D$ con un factor que depende del modo. Si se aplica la unidad para el primer modo, debe tomarse 3.2 y 5.5 para el segundo y tercer modo respectivamente. Este factor empírico coincide, en primera aproximación, con el factor correctivo $g$.

\section{CONCLUSIONES}

La comparación entre el comportamiento dieléctrico y elástico solo se puede realizar si se describen mediante magnitudes comunes. La opción elegida ha sido el comparar, para un mismo valor medio de $D$, los incrementos relativos de los coeficientes $\varepsilon$ y s, así como la energía perdida a lo largo de un ciclo. De entrada, se ha observado un comportamiento cualitativamente distinto entre los materiales PZT blandos y duros. Para un mismo material, se observa una semejanza entre las funciones que describen las alteraciones de los coeficientes $s$ y $\varepsilon$, así como entre las pérdidas de uno y otro tipo. En el primer caso hay concordancia cuantitativa. Ello es explicable si en la no linealidad solo intervienen apreciablemente las paredes de no- $180^{\circ}$. Sin embargo, las pérdidas elásticas son notablemente mayores que las pérdidas dieléctricas, lo que aún no ha podido ser explicado.

Se pueden analizar diversos factores a tener en cuenta en esta comparación, como son el tipo de no linealidad, la distribución de las orientaciones de los dominios, o la falta de homogeneidad del campo. Ello puede permitir relacionar el comportamiento no lineal con la función de distribución (que define el estado de la cerámica) o la interpretación del comportamiento de los modos superiores.

\section{AGRADECIMIENTOS}

Este trabajo ha sido posible gracias a la ayuda del MCYT, dentro del proyecto MAT2001-2325, así como al apoyo de la red europea POLECER (G5RT-CT-2001-05024).

\section{BIBLIOGRAFÍA}

1. P.J. Stevenson, D.A. Hall. "The effect of grain size on the high field dielectric properties of hard PZT ceramics." Ferroelectrics 223, 309-318 (1999).

2. J.E. García, R. Pérez, A. Albareda. “High electric field measurement of dielectric constant and losses of ferroelectric ceramics." J. Phys. D: Appl. Phys. 34, 3279-3284 (2001).

3. D. Damjanovic, G. Robert. "Piezoelectric nonlinearity and Hysteresis in Ferroelectric Materials." Piezoelectric materials for the end user (Conference notes). Edited by N. Setter. Interlaken (2002).

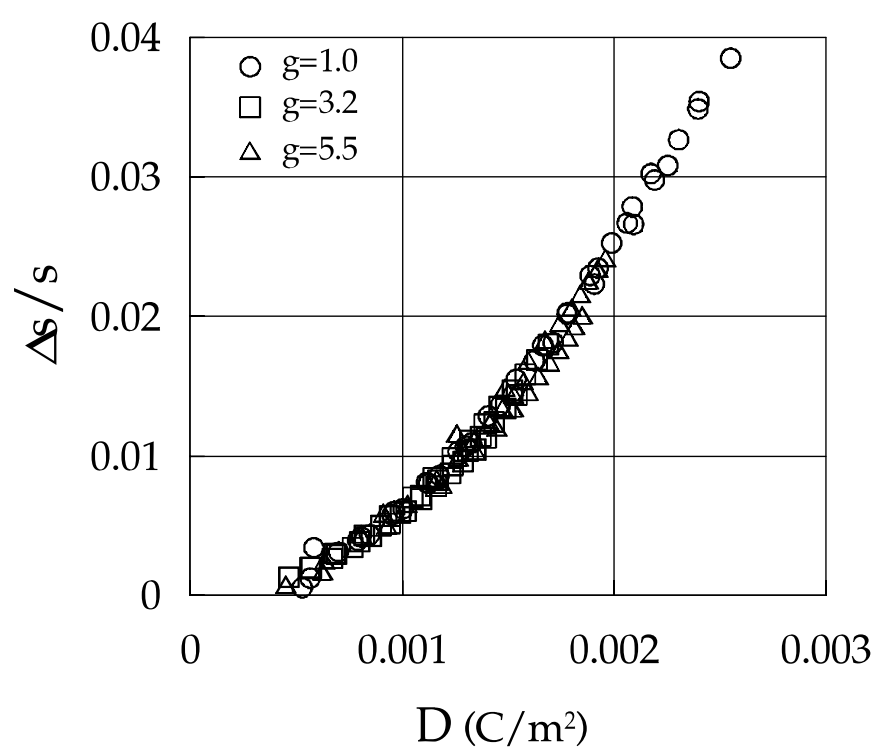

Figura 7: Alteración relativa de $s$ en función de $D$ media para los tres primeros modos radiales. Los resultados presentados se han obtenido a tres frecuencias distintas próximas a la resonancia, para cada modo. Los valores de $D$ han sido escalados según un cierto factor $g$ dependiente del modo, a fin de mostrar su parecido.

4. P. Gonnard. "Piezoelectric material for high power applications: electromechanical characterization and models." Piezoelectric materials for the end user (Conference notes). Edited by N. Setter. Interlaken (2002).

5. R. Perez, A. Albareda. "Analisys of nonlinear effects in a piezoelectric resonator." J. Acoust. Soc. Am., 100, 3561-3569 (1994).

6. K. Uchino. "Recent trend of piezoelectric actuator developments." Proc. ACTUATOR, P34 (2000).

7. B. Andersen, E. Ringaard, T. Bave, A. Albareda, R. Perez. “Performance of piezoelectric ceramic multilayer components based on Hard and soft PZT." Proceedings of the 7th International Conference on New Actuators (Actuator 2000), edited by H. Borgmann (Messe Bremen), 419-422 (2000).

8. W.S. Kreher, J. Rödel. "Disipative domain wall motion and macroscopic response of ferroelectric ceramics." Ferroelectrics 269, 51-56 (2002).

9. D. Damjanovic, M. Demartin. "The Rayleigh law in piezoelectric ceramics." J.Phys. D: Appl.Phys. 29, 2057-2060 (1996).

10. G. Robert, D. Damjanovic, N. Setter, A.V. Turik. "Preisach modeling of piezoelectric nonlinearity in ferroelectric ceramics." J. Appl. Phys. 89, 5067-5075 (2001).

11. S. Li, W. Cao, L.E. Cross. "The Extrinsic nature of Nonlinear behavior observed in lead zirconate titanate ferroelectric ceramic." J. Appl. Phys. 69, 72197224 (1991)

12. G. Arlt, P. Sasko. "Domain configuration and equilibrium size of domains in BaTiO ${ }_{3}$ ceramics." J. Appl. Phys. 51, 4956-4960 (1980).

13. U. Robels, G. Arlt. "Domain wall clamping in ferroelectrics by orientation of defects." J. Appl. Phys. 73, 3454-3460 (1993).

14. W.T. Lee, E.K.H. Salje, U. Bismayer. "Domain-wall structure and domainwall strain." J. Appl. Phys. 93, 9890-9897 (2003).

15. A. Albareda, P. Gonnard, V. Perrin, R. Briot, D. Guyomar. "Characterization of the mechanical nonlinear behavior of piezoelectric ceramics." IEEE Trans. Ultrason. Ferroelectr. Freq. Control 47 844-853 (2000).

16. A. Albareda, R. Perez, J.E. Garcia, J.A. Casals. "No linealidad del comportamiento elástico de las cerámicas piezoelécricas." Bol. Soc. Esp. Ceram. V. En prensa 2003.

17. J.A. Casals, A. Albareda, R. Pérez, J.E. García, E. Minguella, F. Montero de Espinosa. "Nonlinear characterization with burst excitation of 1-3 piezocomposites transducers." Ultrasonic 41, 307-311 (2003).

18. R. Perez, J.E. Garcia, A. Albareda. "Relation Between nonlinear dielectric behavior and alterations of domain structure in a piezoelectric ceramic." Ferroelectrics 273, 205-210 (2002).

19. J.E. Garcia, R. Perez, A. Albareda. “Manifestación de la estructura de dominios en el comportamiento dieléctrico no lineal de una cerámica pierzoeléctrica." Bol. Soc. Esp. Ceram. V. 41, 75-79 (2002).

20. O. Boser. "Statistical Thery of hysteresis in ferroelectric materials." J. Appl. Phys. 62, 1344-1348 (1987). 